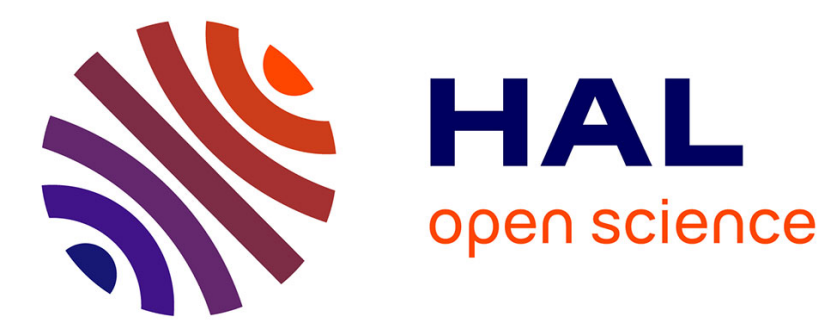

\title{
A numerical model for durotaxis
}

Filippo Stefanoni, Maurizio Ventre, Francesco Mollica, Paolo A. Netti

\section{To cite this version:}

Filippo Stefanoni, Maurizio Ventre, Francesco Mollica, Paolo A. Netti. A numerical model for durotaxis. Journal of Theoretical Biology, 2011, 280 (1), pp.150. 10.1016/j.jtbi.2011.04.001 . hal00701256

\section{HAL Id: hal-00701256 \\ https://hal.science/hal-00701256}

Submitted on 25 May 2012

HAL is a multi-disciplinary open access archive for the deposit and dissemination of scientific research documents, whether they are published or not. The documents may come from teaching and research institutions in France or abroad, or from public or private research centers.
L'archive ouverte pluridisciplinaire HAL, est destinée au dépôt et à la diffusion de documents scientifiques de niveau recherche, publiés ou non, émanant des établissements d'enseignement et de recherche français ou étrangers, des laboratoires publics ou privés. 


\section{Author's Accepted Manuscript}

A numerical model for durotaxis

Filippo Stefanoni, Maurizio Ventre, Francesco Mollica, Paolo A. Netti

PII: S0022-5193(11)00191-3

DOI: doi:10.1016/j.jtbi.2011.04.001

Reference: YJTBI 6432

To appear in: $\quad$ Journal of Theoretical Biology

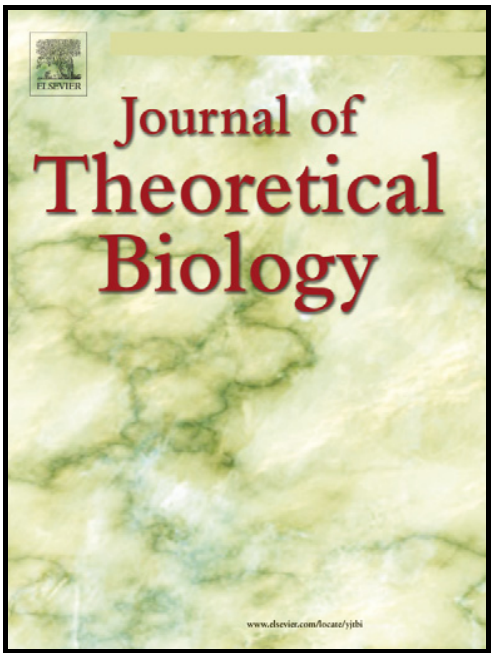

www.elsevier.com/locate/yjtbi

Received date: 29 October 2010

Revised date: 25 March 2011

Accepted date: $\quad 2$ April 2011

Cite this article as: Filippo Stefanoni, Maurizio Ventre, Francesco Mollica and Paolo A. Netti, A numerical model for durotaxis, Journal of Theoretical Biology, doi:10.1016/j.jtbi.2011.04.001

This is a PDF file of an unedited manuscript that has been accepted for publication. As a service to our customers we are providing this early version of the manuscript. The manuscript will undergo copyediting, typesetting, and review of the resulting galley proof before it is published in its final citable form. Please note that during the production process errors may be discovered which could affect the content, and all legal disclaimers that apply to the journal pertain. 
Stefanoni et al.

A Numerical Model for Durotaxis

\section{A Numerical Model For Durotaxis}

Filippo Stefanoni, ${ }^{a}$ Maurizio Ventre, ${ }^{b c}$ Francesco Mollica $^{a}$ and Paolo A. Netti ${ }^{* b c}$

${ }^{\text {a }}$ Department of Engineering, University of Ferrara, Via Saragat 144122 Ferrara, Italy

b IIT@CRIB, Center for Advanced Biomaterials for Healthcare, P.le Tecchio 80, 80125 Naples, Italy

${ }^{\mathrm{c}}$ Interdisciplinary Research Centre on Biomaterials, University of Naples Federico II, P.le Tecchio 80, 80125 Naples, Italy

* Corresponding author: Tel: +390817682408, Fax: +39081676569, email: nettipa@unina.it

\section{ABSTRACT}

Cell migration is a phenomenon that is involved in several physiological processes. In the absence of external guiding factors it shares analogies with Brownian motion. The presence of biochemical or biophysical cues, on the other hand, can influence cell migration transforming it in a biased random movement. Recent studies have shown that different cell types are able to recognize the mechanical properties of the substratum over which they move and that these properties direct the motion through a process called durotaxis. In this work a 2D mathematical model for the description of this phenomenon is presented. The model is based on the Langevin equation that has been modified to take into account the local mechanical properties of the substratum perceived by the cells. Numerical simulations of the model provide individual cell tracks, whose characteristics can be compared with experimental observations directly. The present model is solved for two important cases: an isotropic substratum, to check that random motility is recovered as a sub-case, and a biphasic substratum, to investigate durotaxis. The degree of agreement is satisfactory in both cases. The model can be a useful tool for quantifying relevant parameters of cell migration as a function of the substratum mechanical properties. 
Stefanoni et al.

A Numerical Model for Durotaxis

Keywords: Cell migration, Modelling, Langevin equation, Finite element method 
Stefanoni et al.

A Numerical Model for Durotaxis

\section{Introduction}

Cell migration is a relevant phenomenon in many different biological processes such as morphogenesis, inflammatory response, wound healing and tumour metastasis (Lauffenburger and Linderman, 1993; Chicurel, 2002; Ridley et al., 2003).

Cell crawling is the most common mechanism employed by cells (Ananthakrishnan and Ehrlicher, 2007) and even if it is not the only one known (see for example motility in confined geometry, studied by Hawkins et al., 2009), in the present article we will only refer to crawling. From the microscopic point of view, it is started by specific interactions between the cellular receptors and the ligands present in the extracellular environment, which in turn trigger the activation of the cytoskeleton machinery, eventually leading to cell translocation.

In the absence of external signalling, cell migration occurs through a series of steps taken randomly and the process is called "random motility". As a consequence, a single cell will follow a quasi-straight path over short time intervals, while over longer time intervals such a motion exhibits the characteristics of a persistent random walk, i.e. it has a behaviour similar to Brownian motion (Lauffenburger and Linderman, 1993; Walmod et al., 2001). However, it is known that cells are able to feel a certain number of external signals capable of influencing their movement. These are, for example, the presence of a soluble chemical or of a gradient thereof (chemotaxis; Zigmond, 1977, Harris, 1954), a particular distribution of adhesion molecules on the substratum (haptotaxis, Carter, 1967), the presence of an electrical field (galvanotaxis, Robinson, 1985) or a fluid shear stress (mechanotaxis, Li et al., 2002). Among these, chemotaxis is probably the most studied mechanism (Stokes and Lauffenburger, 1991; 
Stefanoni et al.

A Numerical Model for Durotaxis

Stokes et al., 1991), even though by no means it has to be considered the leading one for orchestrating cell movement.

Additional guidance cues provided by physical and structural properties of the extra cellular matrix (ECM), or of the synthetic substratum, are known to affect cell migration (Friedl and Brocker, 2000; Friedl and Wolf, 2003, Ghosh and Ingber, 2007). The stiffness of the substratum itself, for example, is one of these signals: cells are able to recognize the local mechanical properties, and these are able to influence cell motility in that the moving cell seems to be preferentially directed towards the stiffer regions. This phenomenon is called "durotaxis" and was observed for the first time by Lo et al. (2000). The physical mechanism underlying this phenomenon is not completely understood, but according to Lo et al. (2000), guidance comes through the local protrusions of cells that adhere and probe the mechanical properties of the environment: when the adhesion site occurs on a soft region it is weak and unstable, while if it lands on a stiff region it is strong and stable and becomes the leading edge of the cell. This generates a sort of competition between adhesion sites that leads to the bias that gives rise to durotaxis, as during embryonic development and wound healing. Durotaxis has also been suggested to play a role in other processes such as proliferation, apoptosis and cell differentiation (Engler et al., 2006; Nemir and West, 2010).

For better comprehending the general aspects of cell migration, several mathematical models have been developed. These can be grouped in two principal types, namely continuous approaches and discrete approaches. In a continuous approach cell migration is represented by changes in time and space of the local cell concentration. Many of these models are related to models of diffusion (Patlak, 1953; Keller and Segel, 1971, Chauvière et al. 2007, Painter, 2009, Chauvière et al., 2010), but there are 
Stefanoni et al.

A Numerical Model for Durotaxis

also models describing the evolution of the cell density, which could be of different nature (Filbet et al., 2005). However, continuous approaches do not yield individual cell trajectories which, on the other hand, can be obtained using a discrete approach; for a review on this subject the reader is referred to Ionides et al.(2004). Discrete approaches can be either based on the mechanical equilibrium of a single cell considered as a point mass subjected to external forces (Zaman et al., 2005), or on the direct prescription of the cell velocity vector as a weighted balance of vectorial quantities that are thought of influencing cell motion (Dallon et al., 1999). Since the path of each simulated cell can be obtained directly, the discrete approach is useful for a direct comparison with experiments in which individual cell paths are collected, and for checking the validity of specific hypotheses.

Moreover, it is worth mentioning that discrete model can be upscaled to recover continuous models (Turner et al., 2004, Chauviere and Preziosi, 2010).

Different kinds of cell migration have been modelled using the discrete approach: random motility (Zaman et al., 2005; Dickinson and Tranquillo, 1993), haptotaxis (Smith et al., 2004; Dickinson and Tranquillo, 1993), chemotaxis (Tranquillo and Lauffenburger, 1987; Stokes and Lauffenburger, 1991; Jabbarzadeh and Abrams, 2005) and galvanotaxis (Schienbein and Gruler, 1993). Concerning durotaxis modelling, to our knowledge, it has been studied only using the continuous approach by Moreo et al. (2008). In the present work we develop a simple 2D discrete model for durotaxis, through which it is possible to obtain simulated cell paths that are influenced by the substratum mechanical properties. In particular, the substratum stiffness is taken into account by using a procedure that is reminiscent of the probing mechanism that cells use during motion. 
Stefanoni et al.

A Numerical Model for Durotaxis

\section{Model formulation}

\subsection{Cell migration modelling and the Langevin equation}

In the absence of external guidance cues, cell motility is a stochastic process similar to Brownian motion of particles: although the fundamental mechanisms by which cells move are radically different from the thermally originated movement of particles suspended in a fluid, the observation of the trajectories of individual cells migrating on a substratum reveals a striking similarity, suggesting that a related mathematical description might be appropriate (Dunn and Brown, 1987; Stokes et al., 1991; Schienbein and Gruler, 1993; lonides et al., 2004; Selmeczi et al., 2005).

In fact, the Langevin equation, which was introduced by Paul Langevin (1908) to study Brownian motion, is also a very common model that is employed for describing cell migration (Dunn and Brown, 1987; Stokes and Lauffenburger, 1991). The Langevin equation is one of the easiest dynamical stochastic differential equations, its solution is an Ornstein-Uhlenbeck process, that is the simplest type of continuous autocorrelated stochastic process. Letting $\mathbf{x}(t)$ be the position of a cell on the substratum and denoting time with $t$ and the cell mass with $m$, the Langevin equation reads:

$$
m \frac{\mathrm{d}^{2} \mathbf{x}}{\mathrm{d} t^{2}}=-\zeta \frac{\mathrm{d} \mathbf{x}}{\mathrm{d} t}+\mathbf{F}(t)
$$

This equation might be seen as Newton's second law of motion under the assumption that the cell experiences only two forces: $\mathbf{F}(t)$, a stochastic force which is due to all the probabilistic processes affecting cell motility, and $-\zeta \frac{\mathrm{d} \mathbf{x}}{\mathrm{d} t}$, a drag force that represents all the actions that tend to slow cell 
Stefanoni et al.

A Numerical Model for Durotaxis

movement down, with $\zeta$ being the drag coefficient. On a macroscopic scale, $\mathbf{F}(t)$ can be viewed as normal white noise with zero mean and constant power spectrum. Following the work of Doob (1942), in order to avoid requiring too much regularity on $\mathbf{x}(t)$ this equation can be rewritten in incremental form as follows:

$$
\mathrm{d} \mathbf{v}(t)=-\beta \mathbf{v}(t) \mathrm{d} t+\mathrm{d} \mathbf{B}(t)
$$

where $\beta=\zeta / m$ and $\mathbf{v}(t)$ is the cell velocity, i.e. the time derivative of $\mathbf{x}(t)$. The term $\mathrm{d} \mathbf{B}(t)$ is then assumed to be a Gaussian distributed stochastic process with average zero and variance equal to $\alpha \mathrm{d} t$, where $\alpha$ is a constant and $\mathrm{d} t$ is the time increment. Assuming that $\mathrm{dB}(t)$ is independent of the position $\mathbf{x}(t)$ and using the equipartition theorem of energy, the Langevin equation can be solved for the average value of $\mathbf{x}(t)$ (Coffey et al., 1996). Indicating with $E$ the expected-value operator we can thus obtain the function $D^{2}(t)=E\left\{[\mathbf{x}(t)-\mathbf{x}(0)]^{2}\right\}$, which is the mean square displacement (MSD).

Numerical solutions for the Langevin equation are also possible using a random number generator and a stochastic numerical method. Using the stochastic Euler method (Wright, 1974) the equation must be discretised regularly in time with time increments $\Delta t$ which are sufficiently small but finite:

$$
\mathbf{v}(t+\Delta t)-\mathbf{v}(t)=-\beta \mathbf{v}(t) \Delta t+\mathbf{B}(t+\Delta t)-\mathbf{B}(t)
$$

and the solution in terms of velocity is then stepwise constructed for every time instant if the initial velocity is known. The cell position as a function of time, $\mathbf{x}(t)$, can then be obtained by integration with respect to time $t$, knowing the position $\mathbf{x}_{0}$ of the particle at $t=0$ : 
Stefanoni et al.

A Numerical Model for Durotaxis

$$
\mathbf{x}(t)=\mathbf{x}_{0}+\int_{0}^{t} \mathbf{v}\left(t^{\prime}\right) d t^{\prime}
$$

from which the cell trajectory can be easily constructed.

The Langevin equation contains the basic elements of randomness as well as persistence or inertia and thus provides useful information concerning cell motion in the case of random motility (Lauffenburger and Linderman, 1993). Moreover, it can be used to model chemotaxis by adding to the right hand side of Eq. 1 a deterministic vectorial drift term that depends on the position and strength of the chemoattractant, as it was done by Stokes and Lauffenburger (1991). The case of durotaxis, though, is more complex and can hardly be modelled by simply using a vectorial drift term. In fact, as it is well known, the stiffness of a material is not described by a vector but rather by a tensorial quantity (in general it is a fourth order tensor). Therefore, a correction of the Langevin equation with a deterministic vector should not yield meaningful results in the case of durotaxis, except perhaps for very particular substrata.

\subsection{The cell probing mechanism}

In order to model durotaxis successfully, we believe it could be helpful to consider the effective cell behaviour during crawling. Cell motion occurs in a discontinuous manner, i.e. as a sequence of steps separated by a quiescence time (Ananthakrishnan and Ehrlicher, 2007). Before each step is taken, the cell sends membrane protrusions around its body in a few directions and exerts contractile forces on the substratum through them (see Fig. 1). Seemingly, this procedure aims at probing the local stiffness of the substratum: cell-ECM linkages given by focal adhesions are more stable on stiffer regions; in 
Stefanoni et al.

A Numerical Model for Durotaxis

contrast, focal adhesions that land on softer regions are less firmly obtained and less stable (Pelham and Wang, 1997; Choquet et al., 1997). Since this mechanism takes place at every cell step, it inevitably generates a bias that drives the cell away from compliant regions and towards stiff regions. An important point of this phenomenon, then, is that it is based on a deterministic measurement of the substratum mechanical properties that occurs locally, i.e. at the position currently occupied by the cell in motion. However, cell migration would still remain a fundamentally stochastic event: for instance, the cell does not probe each and every direction and moreover random fluctuations can occur in the dynamics of focal complexes that regulate adhesion or in the intracellular signal trafficking that governs the motile sensing and response mechanism (Friedrichs et al., 2007).

The model for durotaxis that we are seeking should take these aspects into account, i.e. a local measurement of the substratum stiffness to choose the direction, yet preserving some elements of randomness. Let us consider the standard Langevin equation in two dimensions and in particular let us look at the stochastic force term (Eq. 2). In a Cartesian coordinate system both scalar components of $d \mathbf{B}(t)$ can be supposed to be independent and have a normal distribution with zero mean and equal variance. If we switch to polar coordinates, the radial and angular components are again independent and follow a Rayleigh distribution and a uniform distribution in the interval $(-\pi, \pi)$, respectively (Papoulis, 1991). The uniform distribution for the angular component in the case of an isotropic and homogeneous substratum is very reasonable: basically it states that the contribution to motion due to the stochastic force in Eq. 2 is equiprobable in every direction. In durotaxis conditions we hypothesize that the stochastic force term should be changed in such a way that its direction have a higher probability of being parallel to the directions of higher local stiffness. The basic idea, then, is to model durotaxis by replacing the probability distribution of the angular component of the stochastic force. 
Stefanoni et al.

A Numerical Model for Durotaxis

\subsection{Implementing the probing mechanism}

A way for constructing the new probability distribution that takes the local substratum stiffness into account can be inspired by the probing mechanism that was described previously. This can be schematized as a mechanical problem: the cell applies a radial distribution of forces on a linear elastic substratum around its perimeter in order to check the local deformation of the substratum. Here, for simplicity, we will assume that the cell is a circle of diameter $d$ and that the forces are uniform and oriented towards the cell centre (denoted with $p$ in Fig. 2). As a result, for a given distribution of forces, local stiffer directions will yield smaller local displacements at the cell perimeter. Denoting these local displacements along the cell border with $U(\theta$ ), as they will depend only on the direction $\theta$ (see Fig. 2), a suitable measure of the local stiffness as a function of $\theta$ can then be chosen as

$$
k_{\mathbf{x}}(\theta)=\frac{1}{U(\theta)} \quad \text { with } \quad U(\theta)=\max \left\{\mathbf{u}(\theta) \cdot \boldsymbol{\rho}(\theta), U_{\min }\right\} \quad \text { and } \quad \theta \in[0,2 \pi]
$$

\section{(5)}

where $\rho(\theta)$ is the radial unit vector that is oriented from the point on the cell border towards the cell centre, and the constant $U_{\min }=10^{-5} d$ is assumed to be the minimum displacement that the cell is able to sense: from a physical point of view, it appears reasonable that displacements that are smaller than the $0.001 \%$ of the cell diameter are neglected; from the mathematical point of view, the introduction of the constant $U_{\text {min }}$ avoids division by zero errors in the numerical scheme.

Basically, Eq. 5 states that a suitable measure for the local stiffness as a function of direction is the reciprocal of the radial displacement component. Notice that the subscript $\bullet_{\mathbf{x}}$ has been used on $k$ to 


\section{Stefanoni et al.}

A Numerical Model for Durotaxis

mean that it represents a local measure of stiffness, as in general it will depend not only on the mechanical properties and the geometry (i.e. shape, constraints) of the substratum but also on the actual position of the cell. Once $k_{\mathrm{x}}$ is known, a suitable probability density function $P_{k}$ can be constructed as follows:

$$
P_{k}(\theta)=\frac{k_{\mathbf{x}}(\theta)}{\int_{0}^{2 \pi} k_{\mathbf{x}}(\xi) \mathrm{d} \xi} \quad \theta \in[0,2 \pi]
$$

in which the denominator is introduced to normalize the probability density. This distribution indeed has the property of having higher values along the directions where the local stiffness is higher. This is the new probability distribution for the angular component of the stochastic force term that will be used in Eq. 2 for modelling durotaxis.

Concerning the radial component, we will simply keep the Rayleigh probability distribution, i.e. the distribution it would have if cell migration occurred as in the standard Langevin equation, thus we are supposing that this quantity is not influenced by durotaxis. The model finally takes the form

$$
d \mathbf{v}(t)=-\beta \mathbf{v}(t) d t+d \tilde{\mathbf{B}}\left(t, k_{\mathbf{x}}(\theta)\right)
$$

where the new stochastic force $d \tilde{\mathbf{B}}$, which depends on the local stiffness through the angular probability distribution given by Eq. 6, has been employed.

The determination of $k_{\mathrm{x}}$ must be performed at every cell step at the position $\mathbf{x}(t)$ occupied by the cell at current time. This is akin to a standard problem in linear solid mechanics: despite its solution might be in 
Stefanoni et al.

A Numerical Model for Durotaxis

general too difficult to obtain analytically, except in very simple cases, its numerical solution is straightforward and therefore it can be conveniently implemented through the Finite Element Method (FEM) once the cell position, the geometry of the substratum and its mechanical properties are known. The details of this implementation are given in Appendix A.

The model we just presented has been solved for two particular cases, namely migration over an homogeneous and isotropic substratum and migration over a biphasic substratum, i.e. a substratum composed of two adjacent isotropic regions possessing different mechanical properties. The first case is useful in order to check that the model includes the standard Langevin equation as a subcase. The second case represents an experimental set up that is typically employed to study durotaxis (Lo et al., 2000).

\section{Results}

In order to compare the predictions of the model with the experimental results we need to introduce a certain number of quantities. One of the most widely used is the already mentioned MSD, that provides information about the average distance travelled by a cell during migration as a function of time. Clearly, the MSD does not contain any information regarding directionality in the cell movement, therefore, in order to complete the characterisation of cell migration, we will introduce two additional quantities similar to those used by Beltman et al. (2009), namely the turning angle $\gamma_{i}$, i.e. the angle between consecutive segments of the cell paths, and the angle of every path segment with respect to a fixed direction (e.g. one of the coordinate axis, we will use the $x$ axis), denoted with $\delta_{i}$. Indicating with $\mathbf{r}_{i}$ the ith cell step, $\gamma_{i}$ and $\delta_{i}$ have the following expressions: 
Stefanoni et al.

A Numerical Model for Durotaxis

$$
\begin{gathered}
\gamma_{i}=\arccos \left(\frac{\mathbf{r}_{i} \cdot \mathbf{r}_{i+1}}{\left\|\mathbf{r}_{i} \cdot \mathbf{r}_{i+1}\right\|}\right), \\
\delta_{i}=\arccos \left(\frac{\mathbf{r}_{i} \cdot \mathbf{e}_{x}}{\left\|\mathbf{r}_{i} \cdot \mathbf{e}_{x}\right\|}\right) .
\end{gathered}
$$

For the sake of clarity these two quantities are pictured in Fig. 3 for a generic cell path: $\gamma_{i}$ is related to the tendency of the cell of moving in a rectilinear fashion, while $\delta_{i}$ represents the direction chosen by the cell at every step. It immediately follows from Eq. 8 that both $\gamma_{i}$ and $\delta_{i}$ belong to the interval $[0, \pi]$. An additional quantity that can be used to describe cell movement quantitatively is the bias speed, $S_{\text {bias, }}$ also employed by Kipper et al. (2007) for characterizing anisotropic cell motility. If cells are subjected to an attractive field which is oriented, say, in the $x$ direction, it is expected that the average $x$ position of the population of cells increases more or less linearly with time $t$, i.e. $E\{x(t)\} \approx S_{\text {bias }} \cdot t$. Therefore $S_{\text {bias }}$ is estimated by fitting the average $x$ position as a function of time $t$ to a line. As a result, $S_{\text {bias }}$ corresponds to the drift velocity in the $x$ direction: in the case of completely random movement one expects $S_{\text {bias }}=0$, if cell motion is indeed biased the bias speed will be significantly different from 0 .

Let us consider first the case of cell migration over an homogeneous and isotropic substratum. It is worthwhile to study this case to make sure that random motility is recovered and that the numerical scheme procedure works properly: in this case the MSD provided by the model will be compared with the one of the standard Langevin equation whose analytic expression was obtained by Doob (1942):

$$
D^{2}(\Delta t)=2 \frac{\alpha}{\beta^{3}}\left(\beta \Delta t-1+e^{-\beta \Delta t}\right)
$$


Stefanoni et al.

A Numerical Model for Durotaxis

In keeping with the experimental work presented by Stokes et al. (1991) for endothelial cells, the migration parameters will be assumed to be $\alpha=23.2 \mu \mathrm{m}^{2} / \mathrm{h}^{3}$ and $\beta=0.15 \mathrm{~h}^{-1}$. Concerning the model prediction, the paths of 50 cells followed for 24 hours were sequentially simulated over an $800 \mu \mathrm{m} \mathrm{x}$ $800 \mu \mathrm{m}$ square region, with the centre of the square region being assumed as the starting point for all the cells. For simplicity, the substratum is assumed to be linearly elastic and isotropic with a Young's modulus of $100 \mathrm{kPa}$ and a Poisson's ratio of 0.2 (Table 1). The cell paths are depicted in Fig. 4, while the comparison between the MSD of the simulated cells and the one from Eq. 10 is shown in Fig. 5. Using these paths, the angles $\gamma_{i}$ and $\delta_{i}$ have been evaluated and are reported in the histograms of Fig. 6 .

Concerning these results, from Fig. 4 it is clear that the cells move in every direction, as expected. Moreover, comparing the curve obtained from the numerical evaluation of the MSD with Eq. 8, we can see that the agreement is very good (Fig. 5). From Fig. 6(a) we note that most of the angles between consecutive segments (i.e. the $\gamma_{i}$ angles) are very small, say, less than $10^{\circ}$ : this means that we are dealing with a persistent random walk. From Fig. $6(\mathrm{~b})$, showing the histogram of the $\delta_{i}$ angles, it is clear that the path segments follow a quasi-uniform distribution, as in the case of Brownian motion. We can then conclude that when cell migration occurs over an homogeneous and isotropic substratum, the model recovers the standard Langevin equation as a particular case.

The second case of interest, the biphasic domain, is typically used to study durotaxis. Here it has been schematized using a square domain $(500 \mu \mathrm{m} \times 500 \mu \mathrm{m})$, composed of two linearly elastic regions having different Young's moduli (Table 2). In particular, we have chosen that the left half of the domain, i.e. for $0 \mu \mathrm{m}<x<250 \mu \mathrm{m}$, is the stiff part, while the right half, $250 \mu \mathrm{m}<x<500 \mu \mathrm{m}$, is the compliant part. The 
Stefanoni et al.

A Numerical Model for Durotaxis

domain size for this case has been reduced with respect to the previous case because the algorithm in this case is more complex and keeping the original size would have required too much time. Before showing the simulations, it can be interesting to check the effect of the biphasic substratum on the probability distribution given by Eq. 6 . As we can see from Fig. 7, the angular component of the stochastic force of Cell \#2 (in red) has a probability distribution that is basically uniform: its position is relatively far from the interface between the two materials, therefore the expected behaviour is similar to random motility. Cell \#1 (in blue), on the other hand, is positioned right on the interface and in fact the angular probability distribution of its stochastic force is much higher at $180^{\circ}$ than in other directions.

Considering this domain, we can see clearly from Fig. 8 that all the cells that were simulated starting from the centre of the domain migrate towards the stiffer region, in qualitative agreement with the experimental findings of Lo et al. (2000). Also in this case the cells follow a persistent walk, since the distribution of the angles between consecutive path segments has a peak near zero, as depicted in Fig. 9(a). From Fig. 9(b) we can see that the $\delta_{i}$ angles are distributed predominantly in the $90^{\circ}-180^{\circ}$ interval and this confirms that the cells do move towards the stiffer region.

In order to provide a direct comparison between the biphasic domain and the case of random motility, the drift speeds in the $x$ direction and the $y$ direction are shown for both cases in Fig. 10. Here we can see that the bias speed, corresponding to the drift speed in the $x$ direction in the case of the biphasic domain, is much less than 0 , it being around $-16 \mu \mathrm{m} / \mathrm{h}$, while the remaining drift speeds are around 0 . The minus sign in the bias speed means that cells are biased towards the left half of the plane, i.e. towards the negative $x$ direction (see Fig. 8). 
Stefanoni et al.

A Numerical Model for Durotaxis

For the biphasic domain case two different simulations have been performed. The first one considered 50 cells starting sequentially from the centre of the substratum and yielded the trajectories that are depicted in Fig. 8, the histograms of $\gamma_{i}$ and $\delta_{i}$ that are reported in Fig. 9 and the results of Fig. 10. The second simulation has been performed in order to make a direct comparison with the experimental observation reported in the work of Lo et al. (2000): the trajectories of four cells have been generated, two starting from the stiffer region and two starting from the more compliant region, and these are shown in Fig. 11.

\section{Discussions}

In the present work, we have introduced a 2D numerical model which is able to predict cell migration in the case of durotaxis. The Langevin equation, that has been extensively used in the literature for modelling various types of cell migration, forms the basis of the model presented in this paper. Although some forms of biased cell migration, such as chemotaxis, can be easily captured by adding a deterministic drift term to the Langevin equation, durotaxis is more complex and must be treated differently. Moreover, even though the directions of maximum local stiffness can be determined in a deterministic way once the cell position is known, the model had to retain some elements of randomness that anyway do characterize the process of cell migration.

For these reasons we found it convenient to implement the measurement of the local stiffness as a modified distribution probability that the angular component of the stochastic force term in the Langevin equation must obey. This distribution, in general, is far from a simple normal or uniform 
Stefanoni et al.

A Numerical Model for Durotaxis

distribution, and moreover it can vary from point to point. As a result, it is basically impossible to perform an analytical study of the statistics of the relevant processes, such as cell position and velocity, therefore these quantities must be obtained through a numerical procedure. This is what has been done for two benchmark cases, namely the simulation of random motility over an isotropic and homogeneous substratum and migration over a biphasic substratum.

After observing that the model gives results that are at least in qualitative agreement with the experimental data known from the literature, we can make some considerations about the hypotheses that were formulated. Despite the model does not require using a linearly elastic constitutive law for the substratum, we assumed such a law for both the substrata simulated in the present paper. A more general viscoelastic, perhaps even nonlinear, law would have been more appropriate, but it must be considered that during the probing phase the cell applies forces on the substratum within a characteristic time scale that is in the range of $100 \mathrm{~ms}$ up to $1 \mathrm{~s}$ (Kress et al., 2007). If such characteristic times are much smaller than the average relaxation times of the substratum, then the hypothesis of an elastic substratum is acceptable. Moreover, it is also assumed that due to the very small forces applied by the cells (Kress et al., 2007; Oliver et al., 1994), the deformations of the substratum are also very small and this leads to the hypothesis of linearity in the elastic response. Needless to say, these assumptions permit to simplify the FEM setting of the problem, and thus to reduce the computing time requested for the numerical solution.

The material constants of the substratum determined through bulk measurements might not be those actually perceived by the migrating cells. This would be true if ligands adhered rigidly to the material without any mediating molecules. However, ligands could be either weakly bound or connected with a 
Stefanoni et al.

A Numerical Model for Durotaxis

flexible tether to the material surface. In this case the stiffness of this ligand-substratum complex should be considered in evaluating what the cells perceive and thus included in the substratum mechanical properties.

The major limitation of the present model is that it is valid only for low cell densities, i.e. when cells are not too close. In fact, if many cells were considered, the stiffness perceived by a single cell would be altered by the contractile forces exerted by the other ones in its close neighbourhood (Lo et al., 2000). Despite this situation can be handled by the present model without too much effort, it is possible to speculate that cell-cell contacts may occur and these are known to influence the migratory behaviour (Platek et al., 2008). Since the multiple and simultaneous events that take place during cell-cell contact are highly complex, it is very difficult to quantify and model such interactions, in fact this limitation is common to all the discrete models published so far (Flaherty et al., 2007). This notwithstanding, a large body of experimental data on cell migration are indeed based on low cell density assays and this model is able to reproduce this situation.

Considering the positive aspects, the model is simple and versatile, so it can be easily implemented for any substratum. In this paper it has been specialised to two relatively simple cases, but it can be adapted also to cases with more complex geometries and materials. Even though it does not describe all the mechanisms that take part at the cell cytoskeleton (DiMilla et al., 1991), it is able to relate the mechanical properties of the substratum to the path followed by a cell migrating over it, yielding the influence of the substratum stiffness on cell migration. The coupling with experimental data will be easy to obtain, because the model gives the same type of output of a cell tracking experiment. 
Stefanoni et al.

A Numerical Model for Durotaxis

The model as presented is not completely predictive, but can still be a useful tool to perform a robust migration analysis of cells moving in condition of durotaxis, on anisotropic and/or inhomogeneous substrata. In order to make it fully predictive, all the model parameters should be measured independently. In fact, only the material constants and possibly the uniform radial force distribution $p$ applied by the cell and the displacement threshold $U_{\min }$ can be evaluated by means of a local mechanical characterization (e.g. AFM, optical tweezers), but the direct measurement of $\alpha$ and $\beta$ cannot be obtained experimentally. An interesting possibility would be their evaluation using an intracellular mechanosensoring model, which takes into account the forces exerted by the cytoskeleton machinery and its kinetics of assembling/disassembling. More and more of such models are appearing in the current literature and the coupling of one of these models with the one described in the present paper is underway and has to be considered as a future working direction.

In addition, the model can be used to study tissue regeneration and reorganization due to cell migration in tissue engineering applications: it is known that fibroblasts migration along straight lines leads to the deposition of aligned collagen fibres (Wang et al., 2003) and thus the model can be seen as a starting point for designing a scaffold that guides cell migration through its mechanical properties, leading to the production of an engineered tissue with a predetermined collagen alignment. 
Stefanoni et al.

A Numerical Model for Durotaxis

\section{Appendix A}

For the numerical algorithm it is useful to non-dimensionalise Eqs. 2 and 4 using the following nondimensional variables:

$$
\begin{aligned}
& \mathbf{V}=\frac{\mathbf{v}}{\sqrt{\alpha / \beta}} \\
& \mathbf{X}=\frac{\mathbf{x}}{\sqrt{\alpha / \beta^{3}}} \\
& \tau=t \beta .
\end{aligned}
$$

Substituting these definitions into Eqs. 2 and 4 we have

$$
d \mathbf{V}(\tau)=-\mathbf{V}(\tau) d \tau+d \hat{\mathbf{B}}\left(\tau, k_{x}(\theta)\right)
$$

and

$$
\mathbf{X}(\tau)=\mathbf{X}_{0}+\int_{0}^{\tau} \mathbf{V}\left(\tau^{\prime}\right) d \tau^{\prime}
$$

in which $d \hat{\mathbf{B}}$ is the dimensionless stochastic force. More specifically, it is a stochastic process whose radial component has the following Rayleigh probability density: 
Stefanoni et al.

A Numerical Model for Durotaxis

$$
x \exp \left(-\frac{x^{2}}{2}\right) H(x)
$$

where we denoted with $H(x)$ the Heaviside step function. A random variable possessing the probability density given by Eq. A6 can be constructed by creating two normally distributed random variables, each one having zero mean and variance unity, and taking the square root of the sum of their squares. The angular component of $d \hat{\mathbf{B}}$, on the other hand, has the probability density given by Eq. 6 that depends on local stiffness through $k_{\mathrm{x}}(\theta)$.

In order to simulate the cell paths, Eqs. A4 and A5 have been solved numerically using the stochastic Euler method combined with the random number generator of MATLAB (The MathWorks, Natick, MA). In particular, velocity and position at the $i$-th time step, $\mathbf{V}_{i}$ and $\mathbf{X}_{i}$, are given by:

$$
\mathbf{V}_{i}=\mathbf{V}_{i-1}(1-\Delta t)+\Delta \hat{\mathbf{B}}_{i-1}
$$

and

$$
\mathbf{X}_{i}=\mathbf{X}_{i-1}+\mathbf{V}_{i} \Delta \tau
$$

Notice that in our scheme the cell velocity is calculated using an explicit method while position is calculated with an implicit method: we found that such a procedure yielded better results in the case of random motility, i.e. in the case where the standard Langevin equation case had to be recovered.

In order to obtain the angular probability distribution for the stochastic force $\Delta \hat{\mathbf{B}}_{i}$, the stiffness $k_{\mathrm{x}}$ must be evaluated at every cell step. This can be done by solving a linear elasticity problem numerically using 


\section{Stefanoni et al.}

A Numerical Model for Durotaxis

the FEM and has also been realized within MATLAB: the domain has been discretised using four node square elements with two degrees of freedom per node; for the case of random motility 10,000 elements have been used while for the biphasic domain case a total of 6,400 elements have been used (the computational algorithm for the biphasic domain is more complex, thus we needed to use less elements). Once the position of the cell is known, a uniform radial force distribution is applied along the circumference representing the cell perimeter. A cell diameter equals to $25 \mu \mathrm{m}$ has been assumed (see Fig. 2), while the uniform force distribution has been assumed to be of the order $p=10^{-5} \mathrm{~N} / \mathrm{m}$ : this value roughly corresponds to about $10^{2}$ point forces applied along the cell perimeter, each force being of the order of $10 \mathrm{pN}$, in agreement with Kress et al. (2007).

Once the displacements are known at the cell perimeter, the stiffness $k_{\mathrm{x}}$ can be evaluated using Eq. 5 and finally the local probability distribution can be obtained through Eq. 6 . This procedure must be repeated for every moving cell and at every cell step. 
Stefanoni et al.

A Numerical Model for Durotaxis

\section{References}

Ananthakrishnan, R., Ehrlicher, A. 2007. The forces behind cell movement. Int. J. Biol. Sci. 3, 303-317.

Beltman, J.B., Maree, A.F.M., de Boer, R.J. 2009. Analysing immune cell migration. Nature Reviews Immunology 9, 789-798.

Carter, S.B. 1967. Haptotaxis and the mechanism of cell motility. Nature. 213, 256-260.

Chauvière, A., Hillen, T., Preziosi, L. 2007. A continuum model for mesenchymal motion in a fibrous network. Netw. Heterogen. Media. 2, 333-357.

Chauvière, A.; Preziosi, L.; Byrne, H. 2010. A model of cell migration within the extracellular matrix based on a phenotypic switching mechanism. Math. Med. Biol. 27, 255-281.

Chauvière, A., Preziosi, L. Mathematical Framework to Model Migration of Cell Population in Extracellular Matrix, in A. Chauvière, L. Preziosi, C. Verdier, Eds., Cell Mechanics: From Single Scale-Based Models to Multiscale Modeling, CRC Press, 2010.

Chicurel, M. 2002. Cell migration research is on the move. Science. 295, 606-609.

Choquet, D., Felsenfeld, D.P., Sheetz, M.P. 1997. Extracellular matrix rigidity causes strengthening of integrin-cytoskeleton linkages. Cell 88, 39-48. 
Stefanoni et al.

A Numerical Model for Durotaxis

Coffey, W.T., Kalmykov, Y.P., Waldron, J.T. The Langevin equation: with applications in physics, chemistry, and electrical engineering. World Scientific, 1996.

Dallon, J.C., Sherratt, J.A., Maini, P.K. 1999. Mathematical modelling of extracellular matrix dynamics using discrete cells: fiber orientation and tissue regeneration. J. Theor. Biol. 199, 449-471.

Dickinson, R.B., Tranquillo, R.T. 1993. A stochastic model for adhesion-mediated cell random motility and haptotaxis. J. Math. Biol. 31, 563-600.

DiMilla, P.A., Barbee, K., Lauffenburger, D.A. 1991. Mathematical model for the effects of adhesion and mechanics on cell migration speed. Biophys. J. 60, 15-37. Erratum in: Biophys. J. 1991. 60, 983.

Doob, J.L. 1942. The Brownian Movement and Stochastic Equations. Ann. Math. 43, 351-369.

Dunn, G.A., Brown, A.F. 1987. A unified approach to analysing cell motility. J. Cell Sci. Suppl. 8, 81-102.

Engler, A.J., Sen, S., Sweeney, H.L., Discher, D.E. 2006. Matrix elasticity directs stem cell lineage specification. Cell 126, 677-689. 
Stefanoni et al.

A Numerical Model for Durotaxis

Filbet, F.; Laurencot, P.; Perthame, B. 2005. Derivation of hyperbolic models for chemosensitive movement. J. Math. Biol. 50, 189-207.

Flaherty, B., McGarry, J.P., McHugh, P.E. 2007. Mathematical models of cell motility. Cell Biochem. Biophys. 49, 14-28.

Friedl, P.; Brocker, E.B. 2000. The biology of cell locomotion within three-dimensional extracellular matrix. Cell. Mol. Life Sci. 57, 41-64.

Friedl, P.; Wolf, K. Tumour-cell invasion and migration: Diversity and escape mechanisms. Nat. Rev. Cancer. 3, 362-374.

Friedrichs, J., Taubenberger, A., Franz, C.M., Muller, D.J.. 2007. Cellular remodelling of individual collagen fibrils visualized by time-lapse AFM. J. Mol. Biol. 372, 594-607.

Ghosh, K., Ingber, D.E. 2007. Micromechanical control of cell and tissue development: implications for tissue engineering. Adv. Drug Deliv. Rev. 59, 1306-1318.

Harris H. 1954. Role of chemotaxis in inflammation. Phys. Rev. 34, 529-562.

Hawkins, R. J.; Piel, M.; Faure-Andre, G.; Lennon-Dumenil, A. M.; Joanny, J. F.; Prost, J.; Voituriez, R. 2009. Pushing off the walls: a mechanism of cell motility in confinement. Phys. Rev. Lett. 102, 058103. 
Stefanoni et al.

A Numerical Model for Durotaxis

Ionides, E.L., Fang, K.S., Isseroff, R.R., Oster, G.F. 2004. Stochastic models for cell motion and taxis. J. Math. Biol. 48, 23-37.

Jabbarzadeh, E., Abrams, C.F. 2005. Chemotaxis and random motility in unsteady chemoattractant fields: a computational study. J. Theor. Biol. 235, 221-232.

Kipper, M.J., Kleinman, H.K., Wang, F.W. 2007. New method for modeling connective-tissue cell migration: Improved accuracy on motility parameters. Biophys. J. 93, 1797-1808.

Keller, E.F., Segel, L.A. 1971. Model for chemotaxis. J. Theor. Biol. 30, 225-234.

Kress, H., Stelzer, E.H.K., Holzer, D., Buss, F., Griffiths, G., Rohrbach, A. 2007. Filopodia act as phagocytic tentacles and pull with discrete steps and a load-dependent velocity. Proc. Natl. Acad. Sci. USA. 104, 1163311638.

Langevin, P. 1908. On the theory of Brownian motion. C.R. Acad. Sci. (Paris) 146, 530-533.

Lauffenburger, D.A., Linderman, J.J. Receptors: Models for binding, trafficking, and signaling. Oxford University Press Inc, 1993.

Li, S., Butler, P., Wang, Y.X., Hu, Y.L., Han, D.C., Usami, S., Guan, J.L., Chien, S. 2002. The role of the dynamics of focal adhesion kinase in the mechanotaxis of endothelial cells. PNAS. 99, 3546-3551. 
Stefanoni et al.

A Numerical Model for Durotaxis

Lo, C.M., Wang, H.B., Dembo, M., Wang, Y.L. 2000. Cell movement is guided by the rigidity of the substrate. Biophys. J. 79, 144-152.

Moreo, P., Garcia-Aznar, JM; Doblare, M. 2008. Modeling mechanosensing and its effect on the migration and proliferation of adherent cells. Acta Biomater. 4, 613-621.

Nemir, S., West, J.L. 2010. Synthetic materials in the study of cell response to substrate rigidity. Ann.

Biomed. Eng. 38, 2-20.

Oliver, T., Lee, J., Jacobson, K. 1994. Forces exerted by locomoting cells. Seminars in Cell Biology. 5, 139147.

Painter, K.J. 2009. Continuous models for cell migration in tissues and applications to cell sorting via differential chemotaxis. B. Math. Biol. 71, 1117-1147.

Papoulis, A. Probability, Random Variables and Stochastic Processes. Third Edition. McGraw-Hill, 1991, pp. $142-146$.

Patlak, C. 1953. Random walk with persistence and external bias. Bull. Math. Byophys. 15, 311-338. 
Stefanoni et al.

A Numerical Model for Durotaxis

Pelham, R.J. Jr, Wang, Y.I. 1997. Cell locomotion and focal adhesions are regulated by substrate flexibility. Proc. Natl. Acad. Sci. USA. 94, 13661-13665.

Platek, A., Hirano, S., Takeichi, M. 2008. Contact-dependent promotion of cell migration by OLprotocadherin-Nap1 interaction. J. Cell Biol. 182, 395-410.

Ridley, A.J., Schwartz, M.A., Burridge, K., Firtel, R.A., Ginsberg, M.H., Borisy, G., Parsons, J.T., Horwitz, A.R. 2003. Cell migration: integrating signals from front to back. Science 302, 1704-1709.

Robinson, K.A. 1985. The responses of cells to electrical fields: a review. J. Cell Biol. 101, 2023-2027.

Schienbein, M., Gruler, H. 1993. Langevin equation, Fokker-Planck equation and cell migration. Bull. Math. Biol. 55, 585-608.

Selmeczi, D., Mosler, S., Hagedorn, P.H., Larsen, N.B., Flyvbjerg, H: 2005. Cell motility as persistent random motion: theories from experiments. Biophys. J. 89, 912-931.

Smith, J.T., Tomfohr, J.K., Wells, M.C., Beebe, T.P., Kepler, T.B., Reichert, W.M.. 2004. Measurement of cell migration on surface-bound fibronectin gradients. Langmuir 20, 8279-8286.

Stokes, C.L., Lauffenburger, D.A. 1991. Analysis of the roles of microvessel endothelial cell random motility and chemotaxis in angiogenesis. J. Theor. Biol. 152, 377-403. 
Stefanoni et al.

A Numerical Model for Durotaxis

Stokes, C.L., Lauffenburger, D.A., Williams, S.K. 1991. Migration of individual microvessel endothelial cells: stochastic model and parameter measurement. J. Cell Sci. 99(Pt 2), 419-430.

Tranquillo, R.T., Lauffenburger, D.A. 1987. Stochastic model of leukocyte chemosensory movement. J.

Math. Biol. 25, 229-262.

Turner, S., Sherratt, J.A., Painter, K.J; Savill, N.J. 2004. From a discrete to a continuous model of biological cell movement. Phys. Rev. E Stat. Nonlin. Soft Matter Phys. 69, 021910.

Walmod, P.S., Hartmann-Petersen, R., Berezin, A., Prag, S., Kiselyov, V.V., Berezin, V., Bock, E. 2001.

Evaluation of individual-cell motility. Methods Mol. Biol. 161, 59-83.

Wang, J.H.C., Jia, F.Y., Gilbert, T.W., Woo, S.L.Y. 2003. Cell orientation determines the alignment of cellproduced collagenous matrix. J. Biomechanics 36, 97-102.

Wright, D. 1974. The digital simulation of stochastic differential equations. IEEE Trans. Automat. Control. $19,75-76$.

Zaman, M.H., Kamm, R.D., Matsudaira, P., Lauffenburger, D.A. 2005. Computational model for cell migration in three-dimensional matrices. Biophys. J. 89, 1389-1397. 
Stefanoni et al.

A Numerical Model for Durotaxis

Zigmond, S.H. 1977. Ability of polymorphonuclear leukocytes to orient in gradients of chemotactic factors. J. Cell Biol. 75, 606-616. 
Stefanoni et al.

A Numerical Model for Durotaxis

\section{FIGURE LEGENDS}

Fig. 1: A cell probing the substratum in its neighbourhood (Dermal bovine fibroblast grown on a PEG hydrogel substratum. Optical micrograph. Bar $=50 \mu \mathrm{m})$.

Fig. 2: The cell schematized as a circle of diameter $d$. The probing mechanism is approximated through a uniform forces distribution $p$ acting in the radial direction. The position of a generic point $P$ on the cell perimeter is completely determined by the angle $\theta$, which is the one that is used to define the direction of the local stiffness $k_{\mathrm{x}}(\theta)$.

Fig. 3: The definition of the angles $\gamma_{i}$ and $\delta_{i}$ that were used to describe cell migration. In particular, $\gamma_{i}$ is the turning angle, i.e. the angle between two consecutive segments in a path; $\delta_{i}$ is the angle between a path segment and a fixed direction (here the $x$ direction).

Fig. 4: Graphical windrose representation of 50 cell trajectories starting from the centre of the isotropic square domain of $800 \mu \mathrm{m} \times 800 \mu \mathrm{m}$ for 24 hours. The trajectories are random and there is not a preferred direction of migration.

Fig. 5: Comparison between the analytic expression of $D^{2}(\Delta t)$ obtained by Doob, and the numerical evaluation of the same quantity from the model in the case of random motility ( 50 cells, $24 \mathrm{~h}$ over a region of $800 \times 800 \mu \mathrm{m}$, time step $9 \mathrm{~min}$ and $\left.\alpha=23.2 \mu \mathrm{m}^{2} / \mathrm{h}^{3}, \beta=0.15 \mathrm{~h}^{-1}\right)$. The error bars stand for the Standard Deviation. 
Stefanoni et al.

A Numerical Model for Durotaxis

Fig. 6: (a) Graphical representation of the number of steps as a function of $\gamma_{i}$ angles for 50 cells on the isotropic substratum (i.e. in the case of random motility). Most of these angles are close to $0^{\circ}$, meaning that the walk is persistent. (b) Graphical representation of the distribution of the $\delta_{i}$ angles evaluated for 50 cells on an isotropic substratum (i.e. in the case of random motility). There are no preferred directions, the distribution is essentially uniform.

Fig. 7: The probability distribution of the angular component of the stochastic force depends on cell position. The blue cell (\#1) is placed at the interface between the softer material (yellow) and the stiffer material (green), therefore its distribution shows higher probability around $180^{\circ}$, i.e. towards the stiff material. The red cell (\#2) is far from the interface or the boundaries, therefore its stochastic force has an angular component with uniform probability distribution.

Fig. 8: Trajectories of 50 cells spreading from the centre of a biphasic square domain of 500x500 $\mu \mathrm{m}$ for 12 hours. All cells move leftwards, i.e. towards the stiffer region of the domain, as expected from the experimental studies of Lo et al. (2000).

Fig. 9: (a) Graphical representation of the number of steps as a function of $\gamma_{i}$ angles for 50 cells on the biphasic substratum. Also in this case the walk is persistent. (b) Graphical representation of the distribution of the $\delta_{i}$ angles evaluated for 50 cells on a biphasic substratum. As cells migrate preferentially on the stiffer region, i.e. on the left part of the substratum, most of the angles are between $90^{\circ}$ and $180^{\circ}$.

Fig. 10: Drift speeds in the $x$ and $y$ direction for the isotropic domain (case of random motility) and for the biphasic domain. Due to durotaxis, the drift speed in the $x$ direction for the case of biphasic domain is 
Stefanoni et al.

A Numerical Model for Durotaxis

around $-16 \mathrm{~mm} / \mathrm{h}$, a value which is significanlty different from 0 . The error bars represent the standard error of the mean.

Fig. 11: Simulated result for the experimental evidence of Lo et al. (2000). Cells starting from the stiffer region (on the left) do not move to the more compliant one; cells starting from the more compliant region (on the right) move towards the stiffer one (the ending point is shown and the moving direction is indicated by the arrows). Notice that the simulation path lengths are different for each trajectory because the corresponding simulation times differ.

\section{TABLES}

\begin{tabular}{|c|c|}
\hline Random Motility Paramete & \\
\hline Young's modulus $E$ [kPa] & 100 \\
\hline Poisson's ratio $v$ & 0.2 \\
\hline Distributed force $p[\mathrm{~N} / \mathrm{m}]$ & $10^{-5}$ \\
\hline Number of cells & 50 \\
\hline Simulation time length [h] & 24 \\
\hline
\end{tabular}

TABLE 1: Parameters used in the case of random motility. 
Stefanoni et al.

A Numerical Model for Durotaxis

Biphasic Domain Parameters

\begin{tabular}{l|l}
\hline Young's modulus $\mathrm{E}_{1}[\mathrm{kPa}]$ & 100 \\
Young's modulus $\mathrm{E}_{2}[\mathrm{kPa}]$ & 1000 \\
Poisson's ratio $v$ & 0.2 \\
Distributed force $p[\mathrm{~N} / \mathrm{m}]$ & $10^{-5}$ \\
Number of cells & 50 \\
Simulation time length $[\mathrm{h}]$ & 12 \\
\hline
\end{tabular}

TABLE 2: Parameters for the solution of the biphasic domain configuration. 
Stefanoni et al.

A Numerical Model for Durotaxis

$>$ In this study we formulated a 2D theoretical model for durotaxis

$>$ The model is based on the Langevin equation

$>$ Simulations ran with the model agree with experimental observations

$>$ The model is useful for direct comparison with cell tracking experiments 


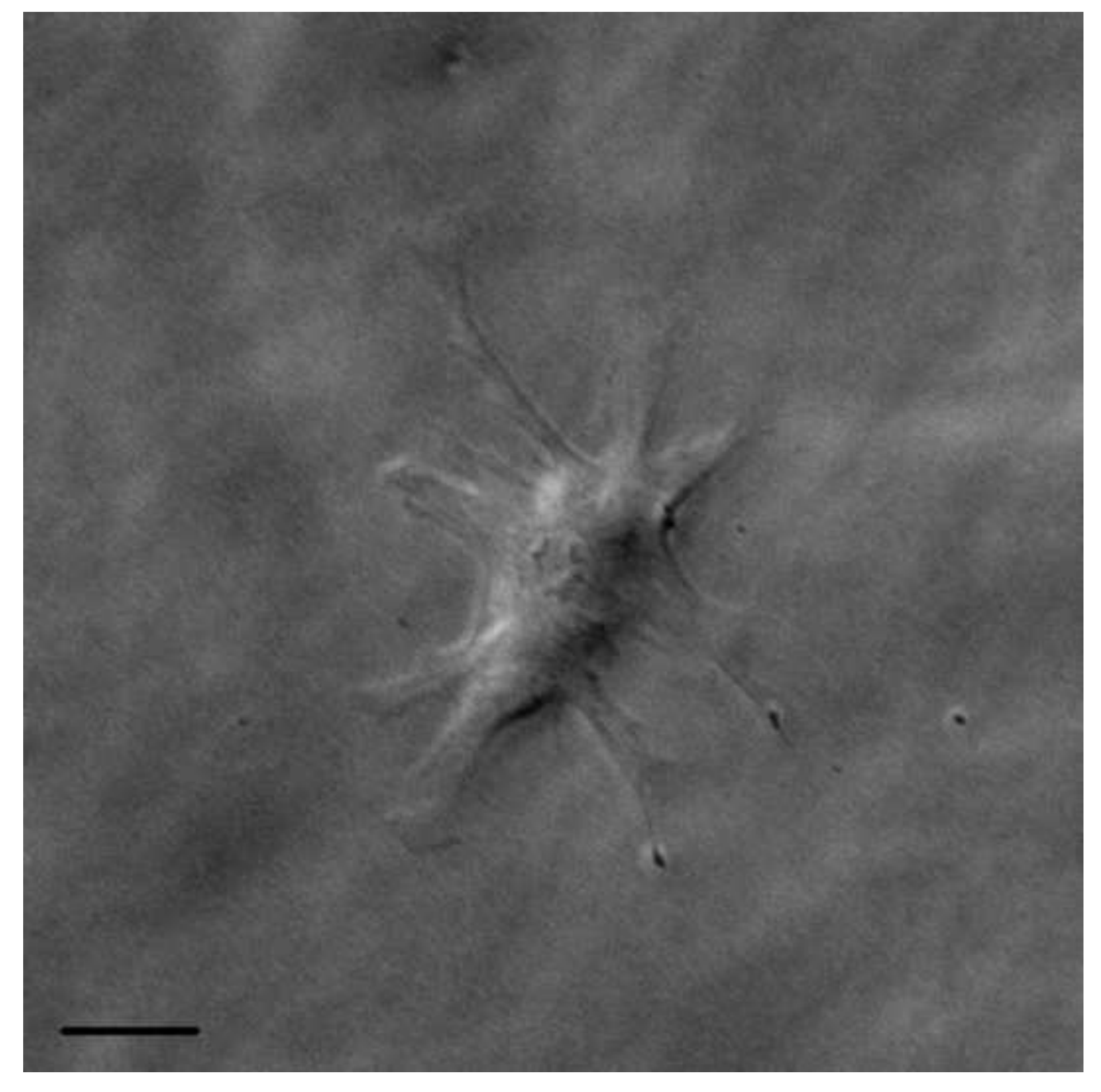

.

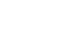

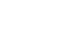

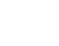

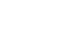
( ( ( . 


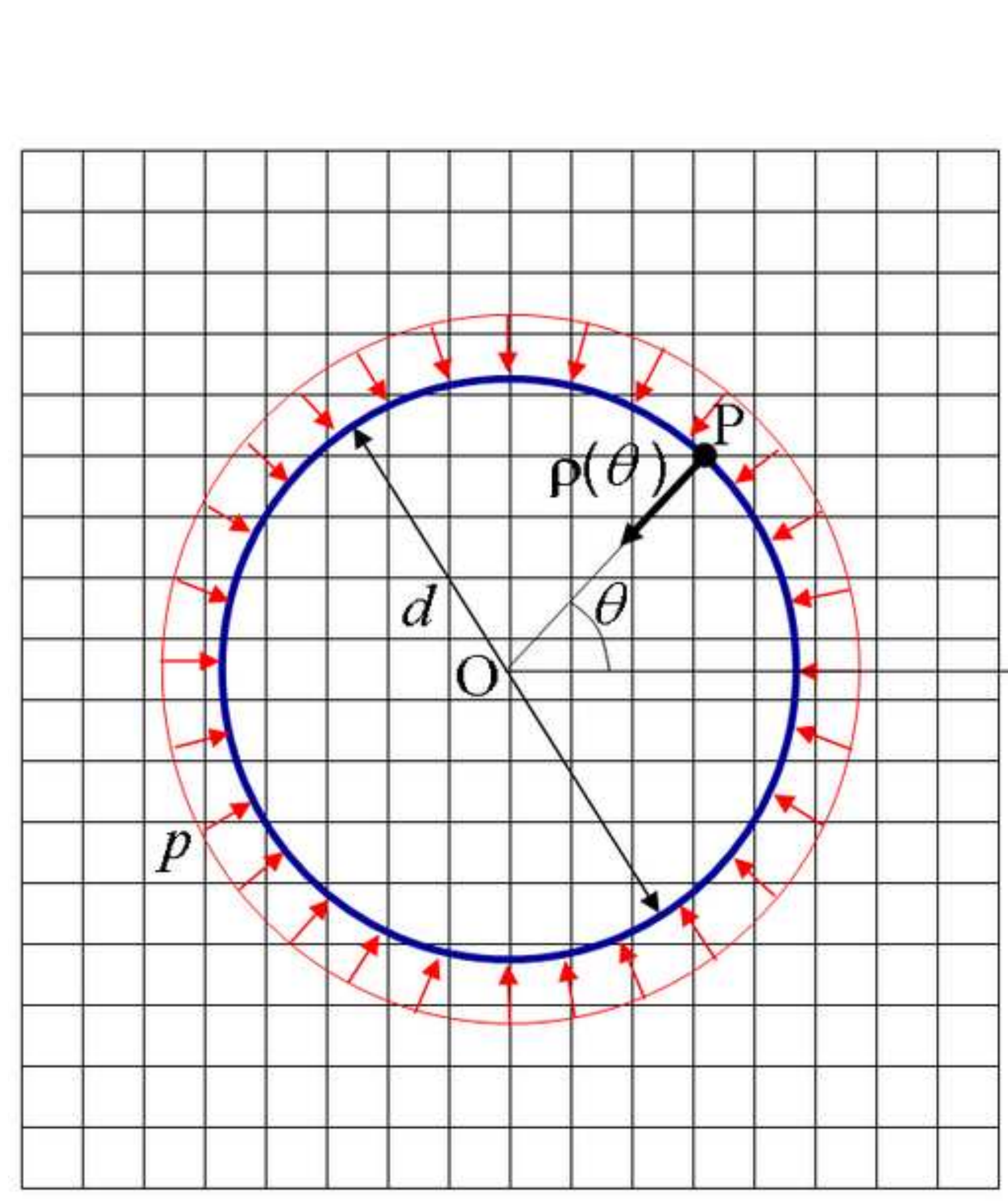



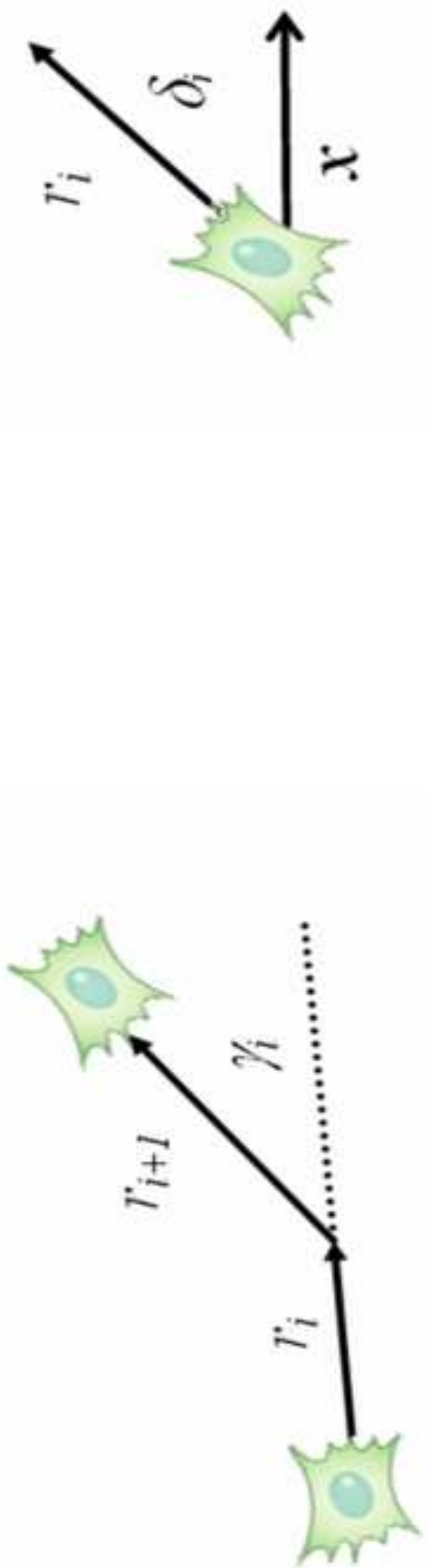

$\stackrel{0}{5}$
$\stackrel{0}{0}$
$\dot{+}$ 


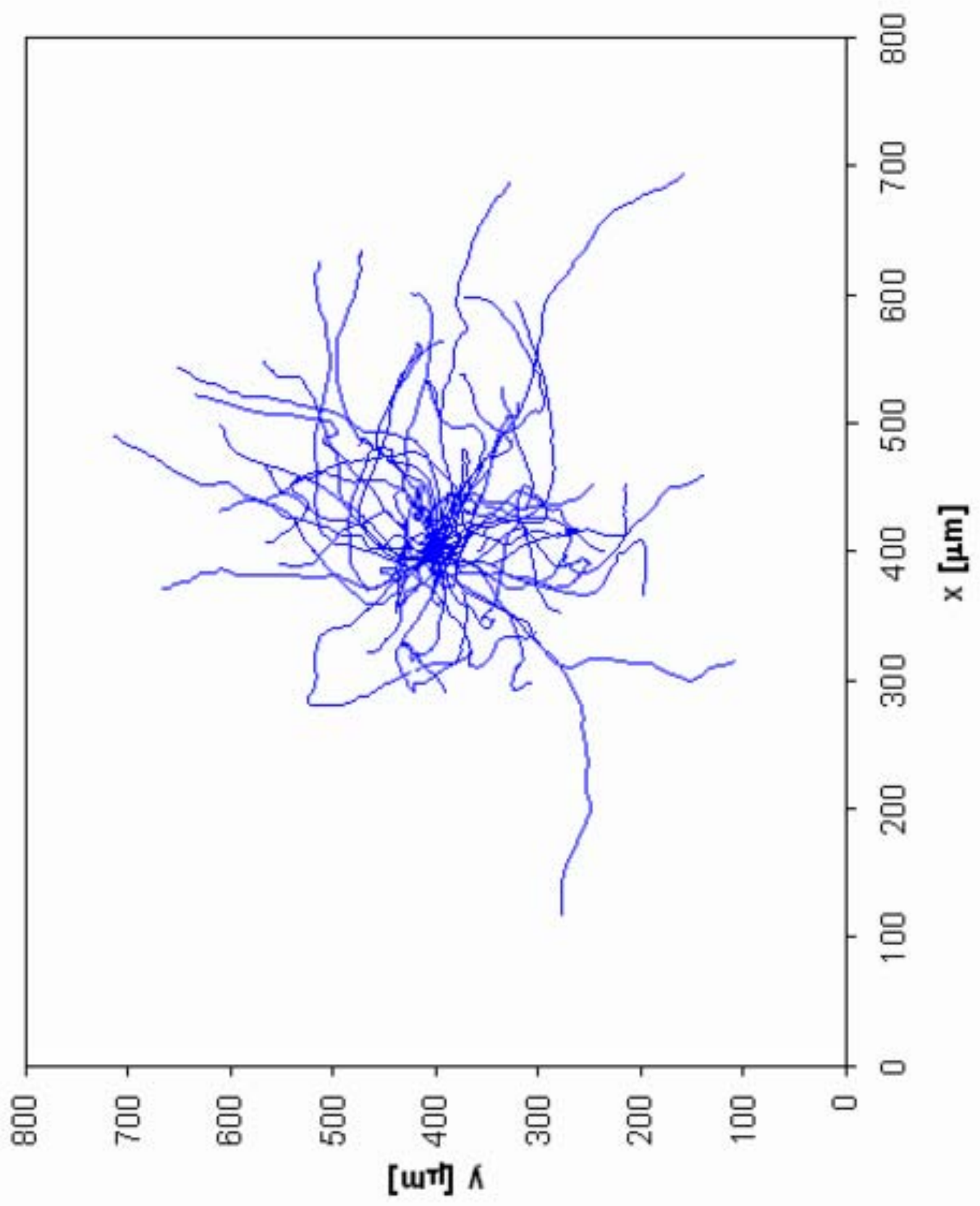




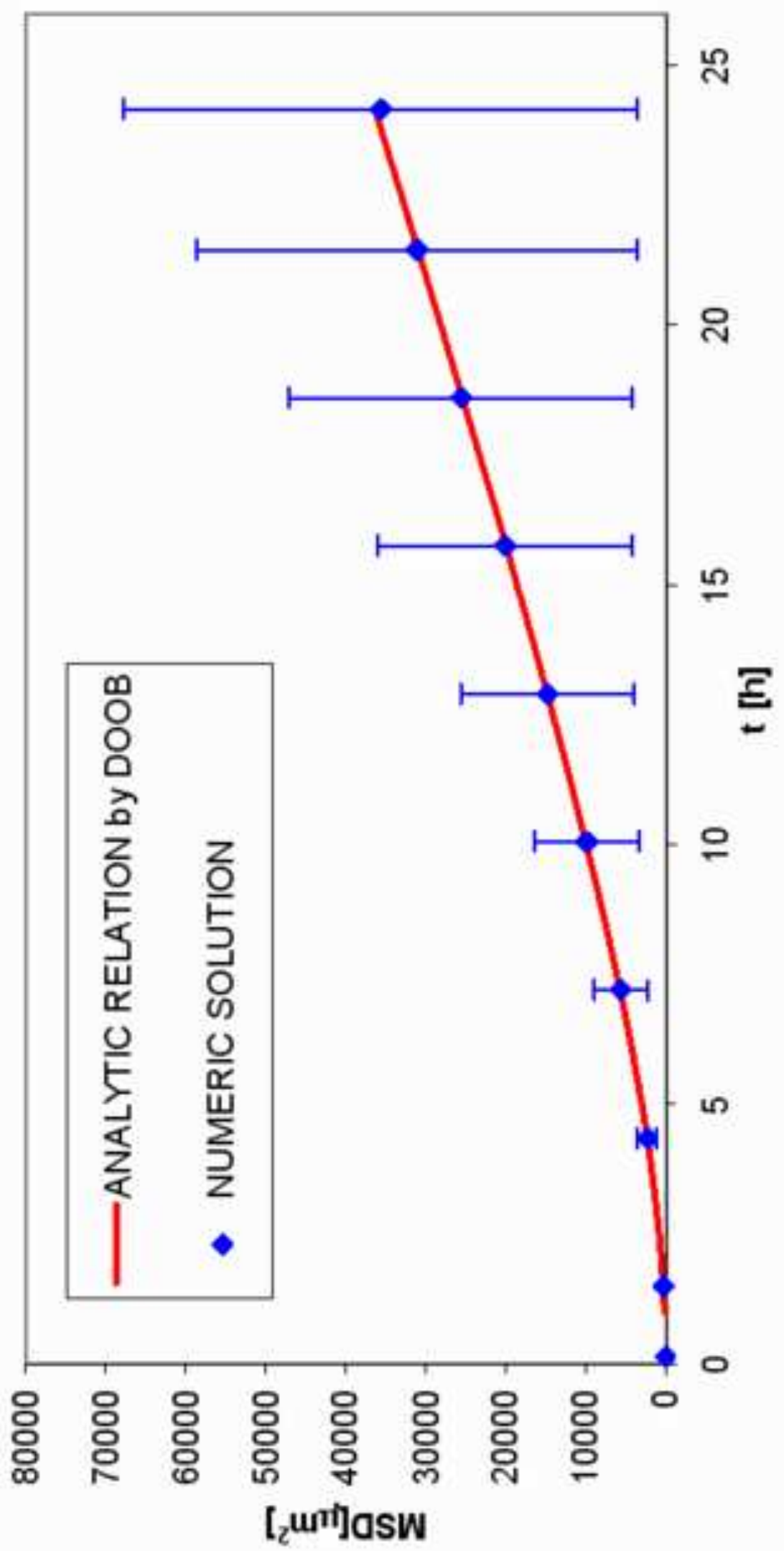



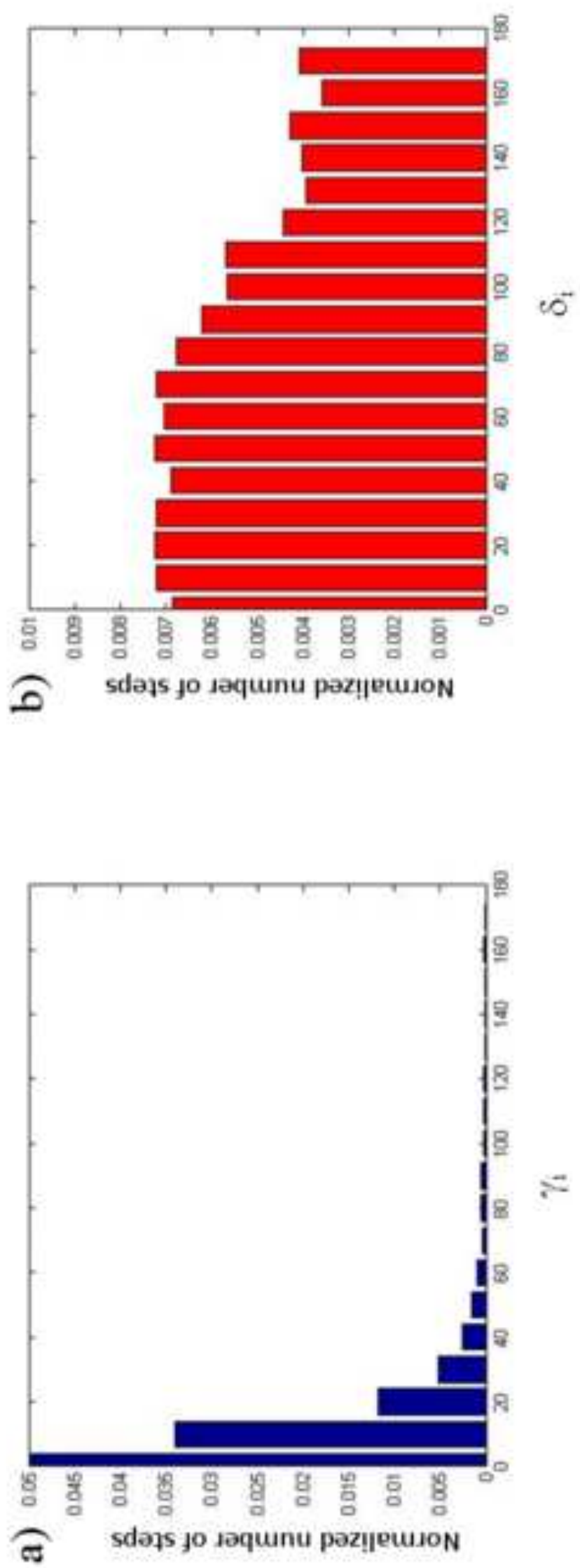

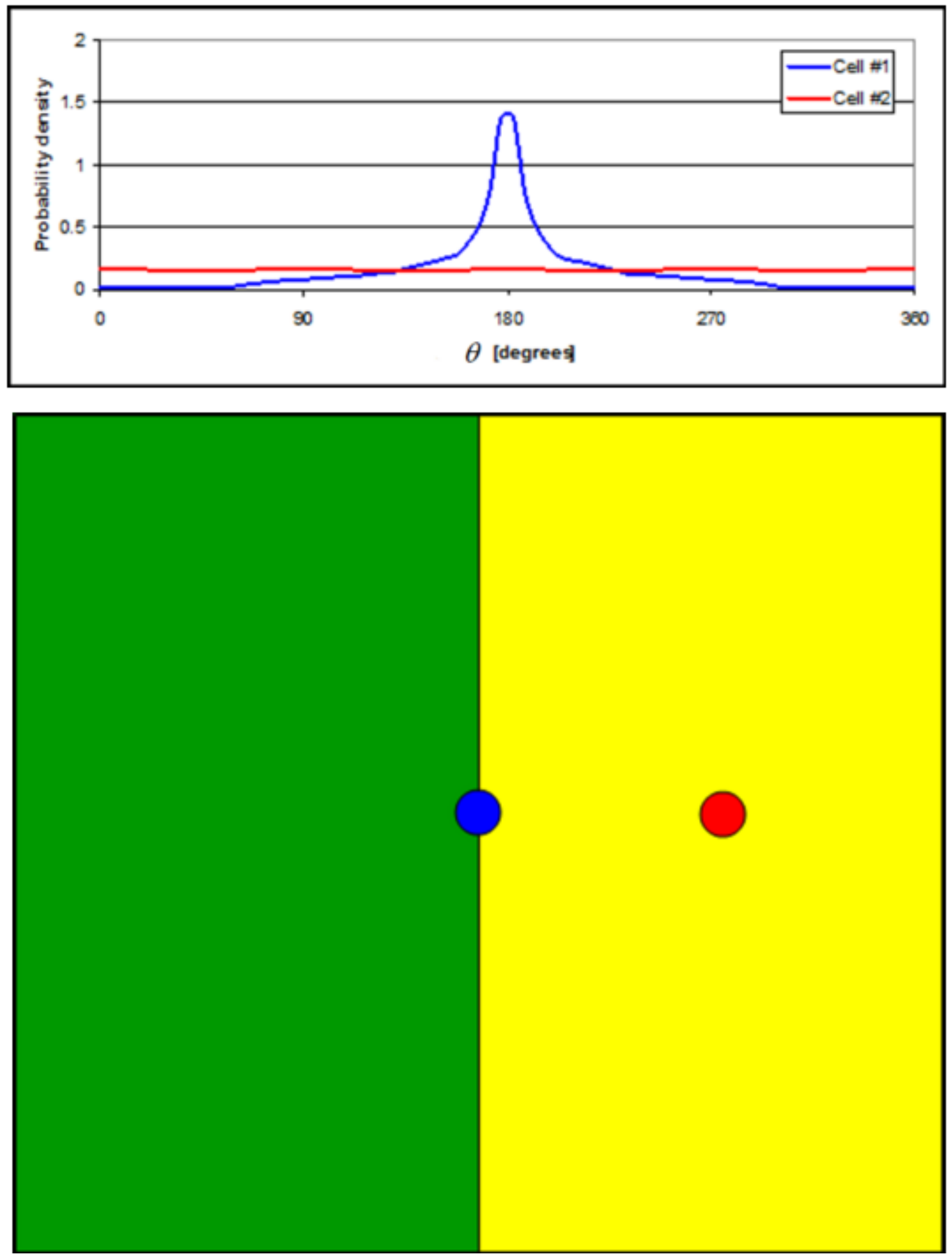


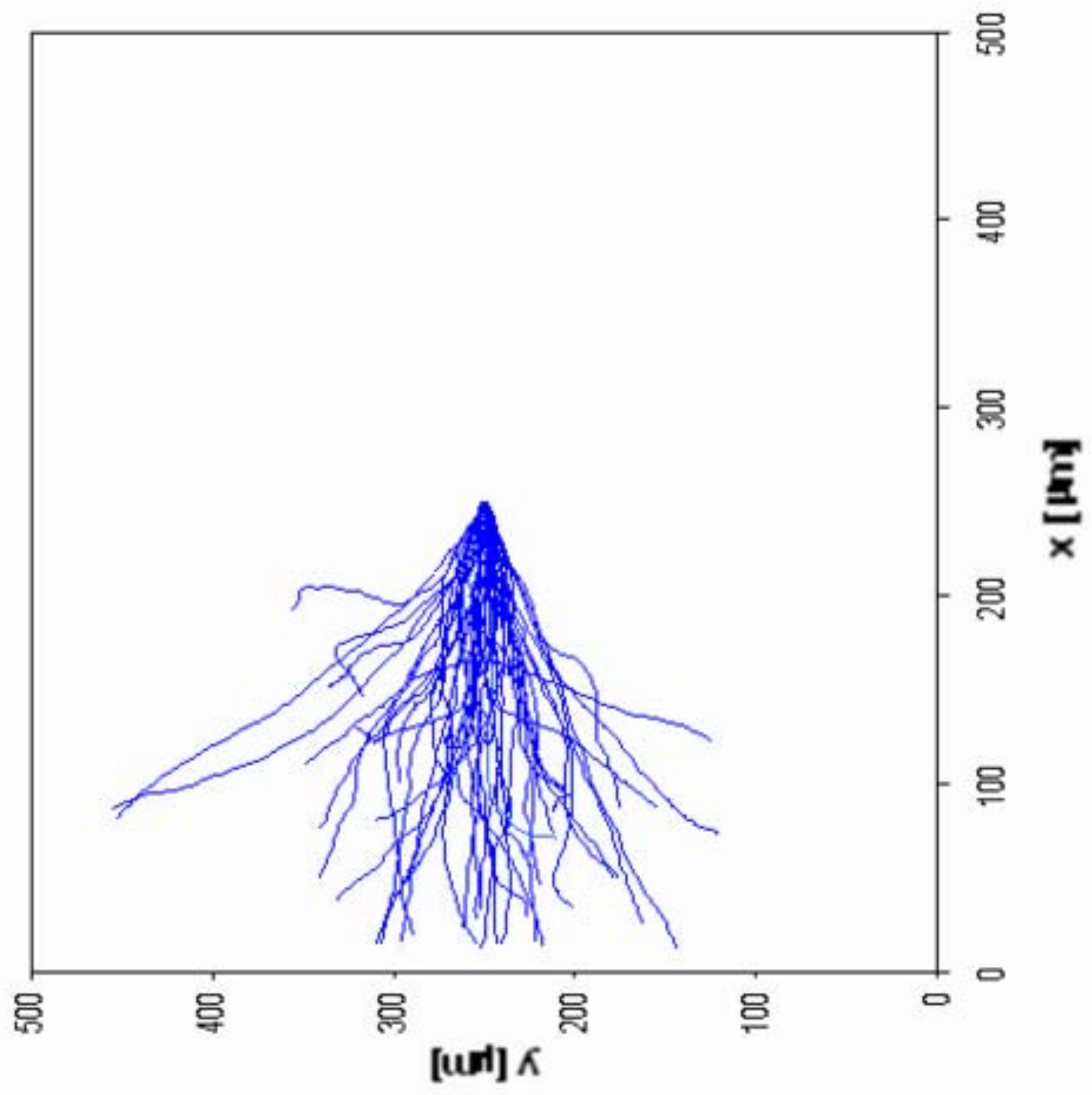



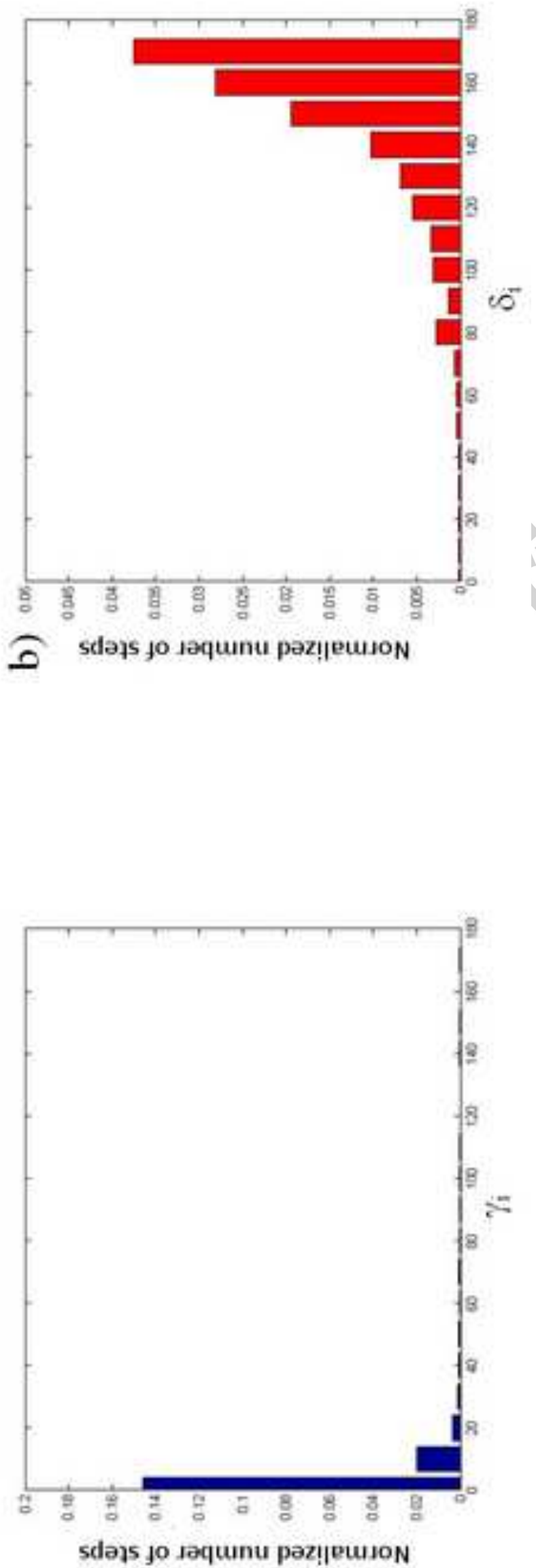

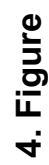




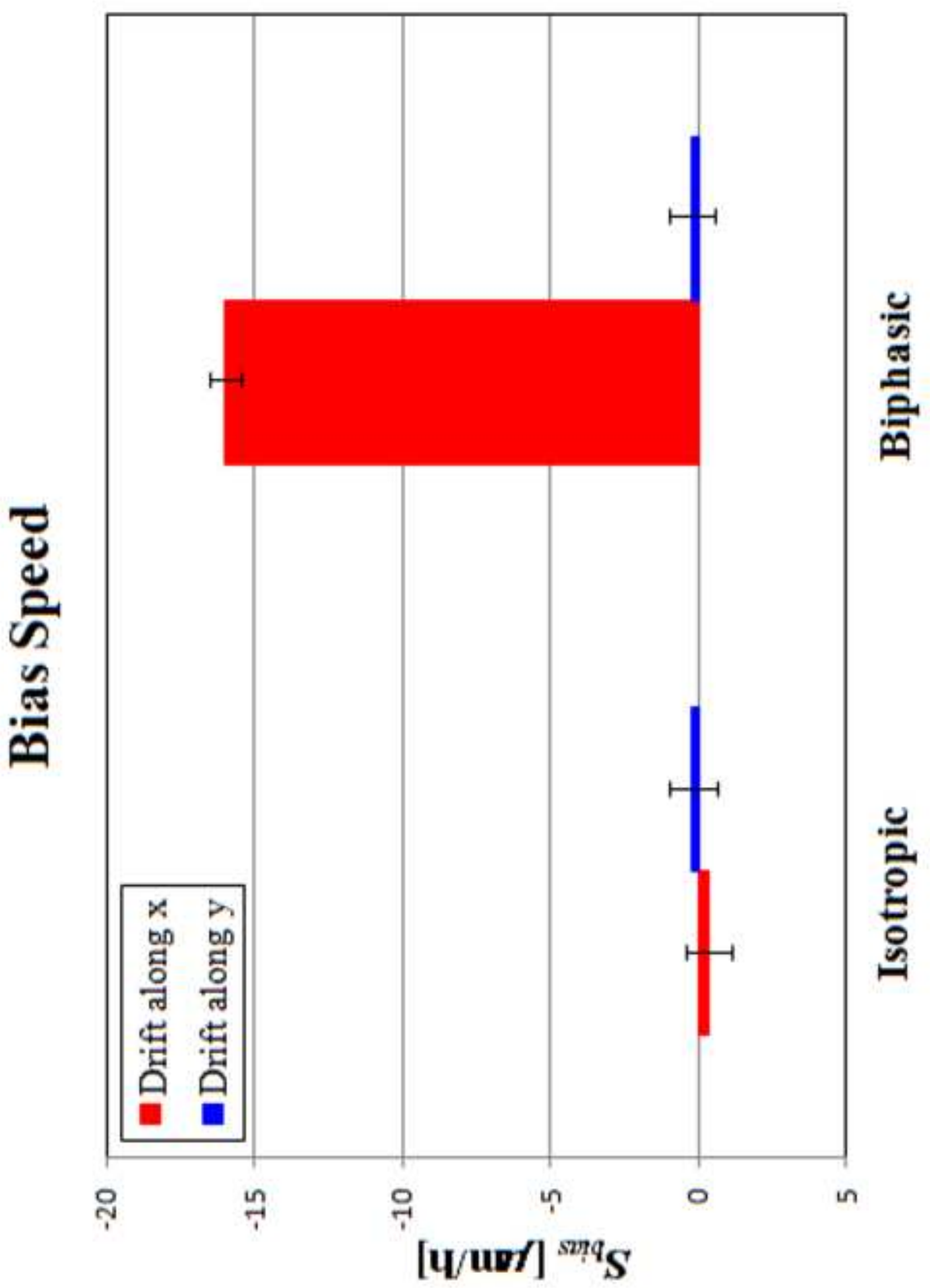

$\frac{0}{2}$
흔
$\dot{+}$ 


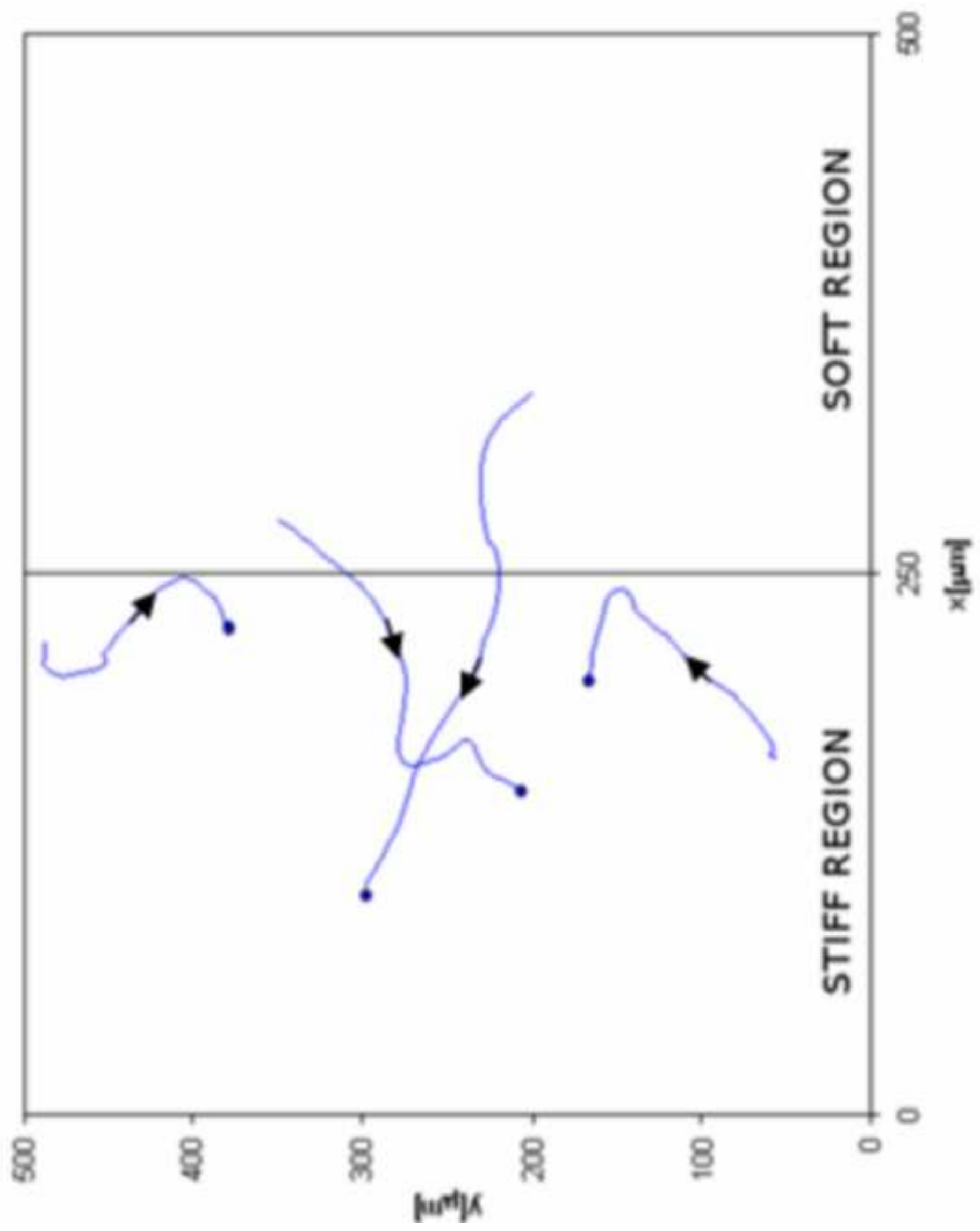

$\underset{\frac{0}{5}}{\frac{0}{2}}$ 